\title{
Intravenous infusion of mesenchymal stem cells promotes functional recovery in a rat model of chronic cerebral infarction
}

\author{
Takahiro Namioka, MD, PhD, ${ }^{1}$ Ai Namioka, MD, PhD, ${ }^{1}$ Masanori Sasaki, MD, PhD, ${ }^{1-3}$ \\ Yuko Kataoka-Sasaki, MD, PhD, ${ }^{1}$ Shinichi Oka, MD, PhD, ${ }^{1}$ Masahito Nakazaki, MD, PhD, ${ }^{1}$ \\ Rie Onodera, PhD, ${ }^{1}$ Junpei Suzuki, PhD, ${ }^{1}$ Yuichi Sasaki, PT, PhD, ${ }^{1}$ Hiroshi Nagahama, PhD, ${ }^{1}$ \\ Jeffery D. Kocsis, PhD, 2,3 and Osamu Honmou, MD, PhD'1-3

\begin{abstract}
${ }^{1}$ Department of Neural Regenerative Medicine, Research Institute for Frontier Medicine, Sapporo Medical University School of Medicine, Sapporo, Japan; '2Department of Neurology, Yale University School of Medicine, New Haven; and ${ }^{3}$ Center for Neuroscience and Regeneration Research, VA Connecticut Healthcare System, West Haven, Connecticut
\end{abstract}

\begin{abstract}
OBJECTIVE Intravenous infusion of mesenchymal stem cells (MSCs) derived from adult bone marrow improves behavioral function in rat models of cerebral infarction. Although clinical studies are ongoing, most studies have focused on the acute or subacute phase of stroke. In the present study, MSCs derived from bone marrow of rats were intravenously infused 8 weeks after the induction of a middle cerebral artery occlusion (MCAO) to investigate whether delayed systemic injection of MSCs improves functional outcome in the chronic phase of stroke in rats.
\end{abstract}

METHODS Eight weeks after induction of the MCAO, the rats were randomized and intravenously infused with either MSCs or vehicle. Ischemic volume and behavioral performance were examined. Blood-brain barrier (BBB) integrity was assessed by quantifying the leakage of Evans blue into the brain parenchyma after intravenous infusion. Immunohistochemical analysis was also performed to evaluate the stability of the BBB.

RESULTS Motor recovery was better in the MSC-treated group than in the vehicle-treated group, with rapid improvement (evident at 1 week post-infusion). In MSC-treated rats, reduced BBB leakage and increased microvasculature/ repair and neovascularization were observed.

CONCLUSIONS These results indicate that the systemic infusion of MSCs results in functional improvement, which is associated with structural changes in the chronic phase of cerebral infarction, including in the stabilization of the BBB.

https://thejns.org/doi/abs/10.3171/2018.5.JNS18140

KEYWORDS transplantation; mesenchymal stem cell; stroke; chronic; vascular disorders

$\mathrm{T}$ HE incidence of stroke, which is the second leading cause of death and the third leading cause of disability worldwide, is expected to increase in the future. ${ }^{4,5}$ Although there have been recent advances in acute reperfusion therapies, including endovascular surgery, which improve clinical outcomes, only a small subpopulation of patients with acute stroke can receive these therapies, and most patients are left with persistent functional disabilities. ${ }^{34}$

A promising cell-based approach in the treatment of cerebral infarction using mesenchymal stem cells (MSCs) is currently being investigated. However, most preclinical studies of MSC therapy have focused on the acute phase of experimental cerebral ischemia. ${ }^{10,14,20-22,27,31,32,36-38}$ The authors of a 2014 meta-analysis concluded, "in preclinical studies, MSCs have consistently improved multiple outcome measures, with very large effect sizes." 32

Acute tissue damage in cerebral infarction results from the interaction of complex pathophysiological cascades, including excitotoxicity mechanisms, inflammatory pathways, oxidative damage, ionic imbalances, and apoptosis. ${ }^{6,7}$ Secondary degeneration occurs several weeks after

ABBREVIATIONS BBB = blood-brain barrier; DMEM = Dulbecco's modified Eagle medium; EvB = Evans blue; $\mathrm{H} \& \mathrm{E}=$ hematoxylin and eosin; i.p. = intraperitoneal; MCA = middle cerebral artery; MCAO = MCA occlusion; MSC = mesenchymal stem cell; NDS = normal donkey serum; PBS = phosphate-buffered saline; PBS-T = PBS $0.1 \%$ and Tween 20; SEM = standard error of the mean.

SUBMITTED January 17, 2018. ACCEPTED May 10, 2018.

INCLUDE WHEN CITING Published online October 26, 2018; DOI: 10.3171/2018.5.JNS18140. 
cerebral ischemia. ${ }^{35}$ Intravenous infusion of MSCs in the acute phase of stroke has been shown to have therapeutic efficacy in animal studies. $13,17,18,27,30$ However, although there are pathophysiological differences between acute and chronic cerebral ischemia, only a limited number of studies have examined the therapeutic efficacy of MSCs during the chronic phase of stroke. Developing approaches to provide therapeutic efficacy in chronic ischemia represents an important area of investigation.

In the present study, MSCs derived from bone marrow were intravenously administered to rats on the 8th week after the induction of middle cerebral artery occlusion (MCAO) to investigate whether delayed systemic injections improved functional outcome in the chronic phase of stroke. In rodent models, endogenous recovery plateaus after approximately 4 weeks, whereas in human patients the initial recovery period is longer and most of their endogenous recovery is completed within the first 3 months after stroke. ${ }^{33}$ Thus, the chosen time period of 8 weeks after MCAO induction in the current study could represent persistent chronic stroke in rodents. Behavioral outcome and histological changes, including the disruption of the blood-brain barrier (BBB), were evaluated to study structural and functional changes after MSC infusion in rats with chronic ischemia.

\section{Methods}

The animal care and use committee of Sapporo Medical University approved the use of animals in the present study; all procedures were conducted in accordance with institutional guidelines. A total of 48 rats were used in this study.

\section{Preparation of MSCs From Rat Bone Marrow}

The MSC culture was prepared according to the methodology used in our previous studies. ${ }^{16,17,27}$ Briefly, bone marrow was obtained from the femoral bones of adult rats $(n=16)$. The marrow was then diluted to $20 \mathrm{ml}$ with Dulbecco's modified Eagle medium (DMEM; Sigma) and supplemented with $10 \%$ heat-inactivated fetal bovine serum (FBS; Thermo Fisher Scientific), $2 \mathrm{mM}$ L-glutamine (Sigma), $100 \mathrm{U} / \mathrm{ml}$ penicillin, and $0.1 \mathrm{mg} / \mathrm{ml}$ streptomycin (Thermo Fisher Scientific) and incubated for 3 days (5\% $\mathrm{CO}_{2}, 37^{\circ} \mathrm{C}$ ). When the cultures had almost reached $100 \%$ confluency, the adherent cells were detached with a trypsin-EDTA solution (Sigma) and subcultured on a $150-\mathrm{mm}^{2}$ Iwaki tissue culture dish (1030-150; surface area $\left.148 \mathrm{~cm}^{2}\right)$ at $5 \times 10^{5}$ cells $/ \mathrm{ml}$ with $14 \mathrm{ml}$ of culture medium. Thus, the plating density was approximately $3.4 \times 10^{3}$ cells $/ \mathrm{cm}^{2}$. Phenotype analysis revealed the following surface antigens on the MSCs: CD45-, ${ }^{-} D 73^{+}, \mathrm{CD} 90^{+}$, and CD106-..12 MSCs were used for in vivo injection at 2 passages.

\section{Cerebral Ischemic Model}

The rat MCAO model was used as a stroke model. We induced permanent unilateral MCAO using a previously reported method of intraluminal vascular occlusion. ${ }^{17,26,27}$ Adult female Sprague-Dawley rats (180-220 g, 9th postnatal week) were anesthetized with an intraperitoneal (i.p.) injection of ketamine $(75 \mathrm{mg} / \mathrm{kg})$ and xylazine $(10 \mathrm{mg} / \mathrm{kg})$.
A length of 20.0-24.0 mm of 3-0 surgical Monosof suture (Medtronic) with the tip rounded by heating near a flame was advanced from the external carotid artery into the lumen of the internal carotid artery until it blocked the origin of the middle cerebral artery (MCA).

\section{Experimental Protocols}

The experimental protocol is shown in Fig. 1. We induced permanent MCAO and obtained T2-weighted MR images to evaluate initial stroke volume after 8 weeks. The rats were then randomized into 2 experimental groups as follows: an MSC-treated group $(\mathrm{n}=12)$ and a vehicle group $(n=12)$. Depending on their group assignment, the rats were intravenously infused with MSCs $\left(1.0 \times 10^{6}\right.$ cells in $1 \mathrm{ml}$ of fresh DMEM) or vehicle alone (1 $\mathrm{ml}$ of fresh DMEM without MSCs) via the left femoral vein. We confirmed high cell viability (> 99\%) with $0.4 \%$ trypan blue immediately after the procedure. All rats were injected daily with cyclosporine A (10 mg/kg, i.p.).

\section{Treadmill Stress Test}

The treadmill stress test was performed according to the following procedure, as in previous studies. ${ }^{17}$ Before the MCAO, rats were trained to run on a motor-driven treadmill (Muromachi Inc.) for $20 \mathrm{~min} /$ day for 2 days a week at a speed of $20 \mathrm{~m} / \mathrm{min}$, with a treadmill slope of $20^{\circ}$. The rats were placed on the moving belt facing away from an electrified grid and induced to run in the direction opposite to the movement of the belt. Thus, the rats had to move forward to avoid foot shocks (1.0-mA intensity). Only rats that had learned to avoid the mild electrical shock were included in the present study. The maximum speed at which the rats could run on a motor-driven treadmill was recorded on day 1 before the injection of MSCs or vehicle, and every week from week 8 through week 16 after the MCAO ( $n=8 /$ MSC group, $n=8 /$ vehicle group). Multiple observers quantified the test results independently to eliminate a source of bias.

\section{MRI Studies and Measurement of Infarct Volume}

MRI was performed as previously reported..$^{17,27}$ Rats were anesthetized with ketamine $(75 \mathrm{mg} / \mathrm{kg})$ and xylazine (10 mg/kg, i.p.) Each rat was placed in an animal holder/ MRI probe apparatus and positioned inside the magnet bore. The animal's head was held in place inside the imaging coil. All MRI measurements were performed using a 7-T, 18-cm-bore superconducting magnet (Oxford Magnet Technologies) interfaced to a Unity INOVA console (Oxford Instruments/Varian Inc.), as previously reported. ${ }^{17,27}$

In brief, T2-weighted coronal images were obtained (TR $3000 \mathrm{msec}$, TE $30 \mathrm{msec}, 1.0-\mathrm{mm}$ slice thickness, 0.5$\mathrm{mm}$ gap, FOV $30 \times 30 \mathrm{~mm}$ ) and reconstructed using a 256 $\times 256$ image matrix. Accurate positioning of the brain was performed to center all slices $5 \mathrm{~mm}$ posterior to the rhinal fissure, with the head of the rat held in a flat skull position. The ischemic lesion area was calculated from T2-weighted images using imaging software (Scion Image, v4.0.2 beta, Scion Corp.), based on the method described in previous reports. ${ }^{19}$ Briefly, the contrast was optimally adjusted. The lesion was defined as the area where the signal intensity 


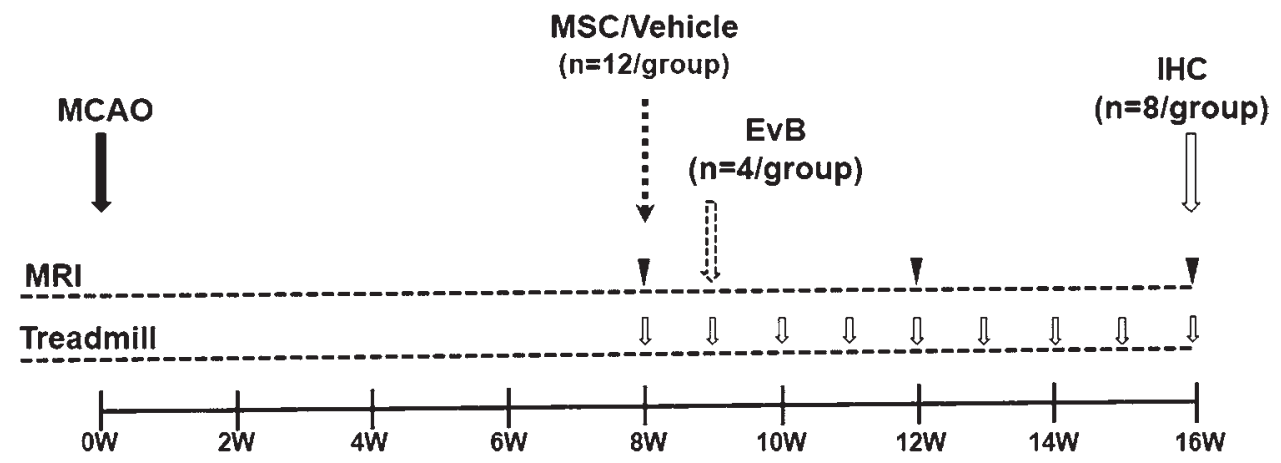

FIG. 1. Experimental protocol. MCAO was induced in 9-week postnatal rats. T2-weighted images obtained 8 weeks after MCAO were used to exclude rats with infarcted volumes $<230 \mathrm{~mm}^{3}$. The rats that were included in the present study were then randomized into the 2 following experimental groups: an MSC-treated group and a vehicle group. The rats were then administered vehicle or MSCs intravenously (8 weeks after MCAO). Thereafter, T2-weighted MR images were obtained at 12 and 16 weeks after MCAO (i.e., 4 and 8 weeks after the infusion, respectively). Results of testing on a motor-driven treadmill were recorded on day 1 before the injection of MSCs or vehicle and every week from 8 weeks through 16 weeks after MCAO. Extravasated EvB analyses were performed at 9 weeks after the induction of the MCAO (1 week after infusion). Immunohistochemical analyses were performed 16 weeks after the MCAO (8 weeks after the infusion). IHC = immunohistochemistry; $W=$ weeks.

was at least 1.25 times that of the corresponding area in the contralateral hemisphere; based on this definition, the lesions completely covered the territory of the occluded MCA in all of the rats. The edges of the area defined by this degree of increase in signal intensity were manually traced on each of the 9 coronal slices. Thereafter, the hyperintense areas were then summed and multiplied by the slice thickness plus the interslice gap to calculate the lesion volume.

T2-weighted MR images obtained 8 weeks after MCAO were used to exclude rats with infarcted volumes below $230 \mathrm{~mm}^{3}$. T2-weighted images were obtained at 12 and 16 weeks after MCAO $(n=8 /$ MSC group, $n=8 /$ vehicle group).

\section{Evans Blue Analysis}

The disruption of the BBB after ischemia was assessed with extravasated Evans blue (EvB) dye (Sigma), as described previously, with minor modifications. Four rats in each group were intravenously infused with MSCs $(n=4)$ or vehicle alone $(n=4), 8$ weeks after MCAO, and then used for EvB analysis 1 week later. The rats were anesthetized with ketamine and xylazine (respectively, 75 and $10 \mathrm{mg} / \mathrm{kg}$, i.p.). Then, $10 \% \mathrm{EvB}$, dissolved in $0.9 \%$ saline, was injected as a single bolus dose of $1 \mathrm{ml} / \mathrm{kg}$, via the right femoral vein. One day after the injection of EvB, the rats were deeply anesthetized with ketamine and xylazine (respectively, 75 and $10 \mathrm{mg} / \mathrm{kg}$, i.p.) and transcardially perfused with ice-cold phosphate-buffered saline (PBS) to remove the intravascular dye. The entire brain of each rat was then removed and dissected into $0.1-\mathrm{mm}$ coronal sections using a vibratome. The coronal slices were examined at $\times 7$ magnification, under a Zeiss AXIO Zoom V16 microscope (Carl Zeiss AG), which was equipped with appropriate laser and filter sets and a high-resolution CCD camera (AxioCam 506 mono; Carl Zeiss). The EvB $(640 / 30 \mathrm{~nm})$ was observed with the appropriate excitation filters. Quantitative analysis of the EvB-extravasated area was performed using the ZEN 2.3 imaging software for microscopy (blue edition; Zeiss). Fluorescence pixel intensities for extravasated and unextravasated EvB areas were computed with ZEN 2.3 blue edition. The EvB-extravasated area was outlined and the sum of the pixel intensities in the EvB-extravasated area was obtained.

\section{Immunohistochemistry}

At 16 weeks after MCAO induction (8 weeks after MSC or vehicle infusion), rats $(n=8 /$ MSC group, $n=8 /$ vehicle group) were deeply anesthetized with an overdose of ketamine $(100 \mathrm{mg} / \mathrm{kg})$ and xylazine $(20 \mathrm{mg} / \mathrm{kg}$, i.p.), before being perfused with $4 \%$ paraformaldehyde following PBS. The rat brains were removed and processed for standard frozen sectioning. Then, $10-\mu \mathrm{m}$-thick cryosections of the brain were cut using a cryostat and mounted on glass slides. Sections were washed in PBS $0.1 \%$ and Tween 20 (PBS-T) 3 times and blocked in 5\% normal donkey serum (NDS) and 0.3\% Triton X-100 in PBS at room temperature for 30 minutes. The sections were then incubated overnight in primary antibodies diluted with $5 \%$ NDS, $0.3 \%$ Triton X-100, and PBS at $4^{\circ} \mathrm{C}$. The cryosections were processed for immunolabeling for mouse monoclonal RECA-1 (1:400; AbD Serotec MCA970R) for the detection of endothelial cells and rabbit monoclonal anti-PDGFR- $\beta$ antibody (1:800; ab32570) for the detection of pericytes. The sections were then washed in PBS-T 4 times before being incubated in secondary antibodies anti-mouse IgG Alexa Fluor 594 for RECA-1 (1:1000; Invitrogen, A11037) and goat anti-rabbit IgG Alexa Fluor 488 conjugate for PDGFR- $\beta$ (1:1000; Invitrogen, A11032), counterstained with DAPI, and coverslipped with Vectashield (Vector Laboratories). The frozen sections were examined using confocal microscopy (LSM780 ELYRA S.1 system).

For assessment of vascular staining for RECA-1 and PDGFR- $\beta$, image analysis was performed similarly to that described previously, with minor modifications. ${ }^{3,16} \mathrm{Im}-$ ages were acquired within the lesion. For capillary length, RECA- $1^{+}$and PDGFR- $\beta^{+}$capillary profiles were measured 


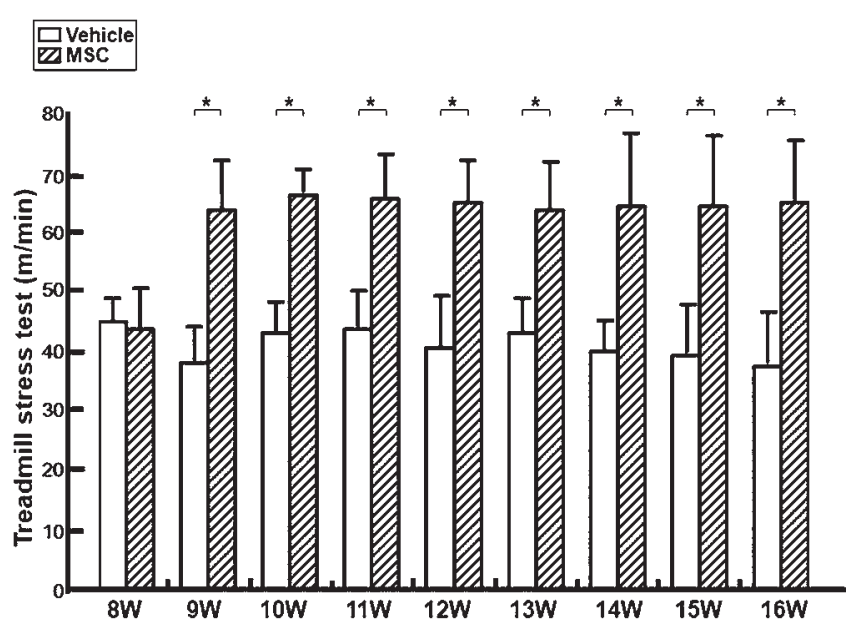

FIG. 2. Treadmill stress test. There was no significant difference between the mean values for the MSC-treated group and vehicle group at 8 weeks after MCAO, prior to the infusion. The maximum treadmill velocity was recorded every week during the 8 weeks after the infusion; the MSC-infused groups had a greater maximum velocity, which peaked at approximately 1 week after MSC infusion. The vehicle group showed no recovery. Data are presented as mean and SEM (error bars). ${ }^{*} p<$ 0.0001 .

using the ImageJ length analysis tool ( $<6 \mu \mathrm{m}$ in diameter). The length of RECA- $1^{+}$and PDGFR- $\beta^{+}$vascular profiles per square millimeter of brain tissue around the infarcted area was expressed in millimeters. The length of capillaries that stained positive for RECA- 1 and PDGFR- $\beta$ was measured at 3 points per sample, and the average of the 4 samples was calculated. Pericyte coverage was determined as a percentage of the PDGFR- $\beta^{+}$pericyte surface area covering the RECA- $1^{+}$capillary surface area per field $(845 \times 845 \mu \mathrm{m})$.

\section{Statistical Analysis}

All statistical analyses were performed using JMP Pro 12.2.0 (SAS Institute Inc.). Comparison of the experimental and control groups was performed using a Student ttest. Significance levels were designated as $p<0.01$ or $p$ $<0.001$. Results of quantitative analyses are reported as mean \pm standard error of the mean (SEM).

\section{Results}

\section{Behavioral Function}

The maximum velocity at which rats could run on a motor-driven treadmill was recorded in the treadmill test. Although the rats reached a velocity of approximately 70 $\mathrm{m} / \mathrm{min}$ before the MCAO, they showed motor deficits after the MCAO; the endogenous recovery reached a plateau with a residual deficit. There were no significant differences between the groups, before vehicle or MSC infusion, at 8 weeks after MCAO induction (45 $\pm 4 \mathrm{~m} / \mathrm{min}$ for the vehicle-treated group vs $44 \pm 6 \mathrm{~m} / \mathrm{min}$ for the MSCtreated group, $\mathrm{p}=0.7532)$. The maximum velocity was recorded every week during the study period (8 weeks after MSC or vehicle infusion). The MSC-infused group had a greater mean maximum velocity than the vehicle group

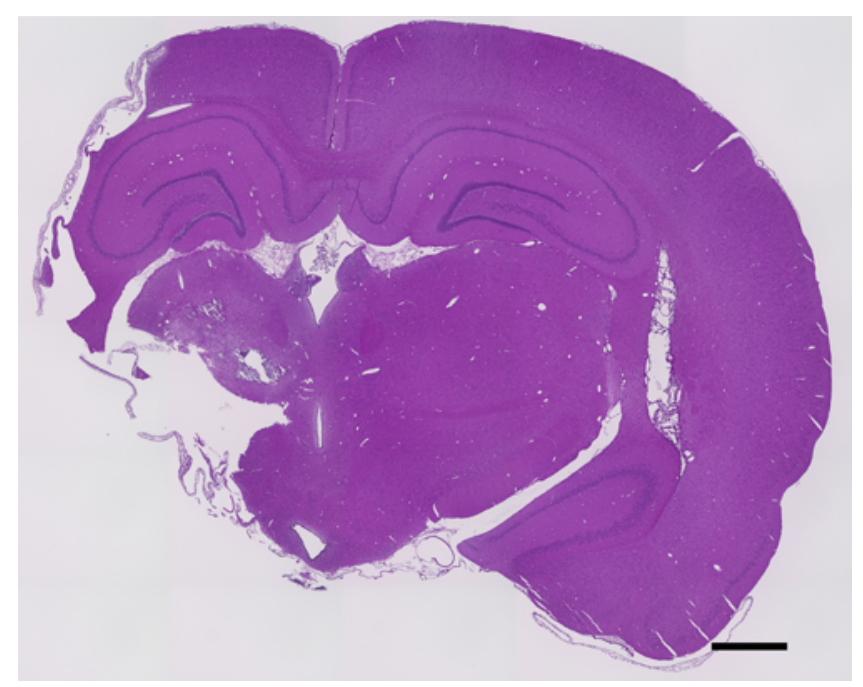

FIG. 3. Photomicrograph of an $\mathrm{H}$ \& E-stained brain section from a rat killed 8 weeks after MCAO induction showing an untreated lesion. Scale bar $=1 \mathrm{~mm}$.

(Fig. 2). The mean values for the vehicle and MSC groups at 1 week after MSC infusion were $38 \pm 6 \mathrm{~m} / \mathrm{min}$ and 64 $\pm 8 \mathrm{~m} / \mathrm{min}$, respectively $(\mathrm{p}<0.0001)$. This difference was maintained at the last test point at 16 weeks after infusion. These results indicate that intravenous administration of MSCs improved functional outcome, beginning at 1 week after infusion. Moreover, this improvement was maintained. Figure 2 summarizes these data.

\section{Ischemic Lesion Volume by MR Image Analysis}

Hematoxylin and eosin ( $\mathrm{H} \& \mathrm{E}$ ) staining at 8 weeks after MCAO induction, before any animals underwent vehicle or MSC infusion, revealed large necrotic cavity formation in the ischemic hemisphere, fibrous gliosis in the wall of the cavity, and infiltration of only a few inflammatory cells around the formed cavity (Fig. 3). Pre-treatment MRI also demonstrated a large area of high signal intensity in the ischemic hemisphere (Fig. 4A and D). The ischemic lesion volume was estimated for both groups using in vivo MRI (Fig. 4). T2-weighted MR images were obtained in animals from the experimental group at 8, 12, and 16 weeks after the MCAO. T2-weighted images obtained 8 weeks after the MCAO, before the injection of MSCs or vehicle, confirmed that there were no differences in the stroke volume between the groups $\left(285.8 \pm 32.7 \mathrm{~mm}^{3}\right.$ in the MSC group vs $284.6 \pm 28.8 \mathrm{~mm}^{3}$ in the vehicle group, $\mathrm{p}=0.9172$ ). The lesion volume for each group was determined by analyzing T2-weighted MR images obtained at 12 weeks $\left(291.1 \pm 38.3 \mathrm{~mm}^{3}\right.$ in the MSC group vs $283.9 \pm$ $28.7 \mathrm{~mm}^{3}$ in the vehicle group, $\mathrm{p}=0.6040$ ) and 16 weeks $\left(294.9 \pm 36.0 \mathrm{~mm}^{3}\right.$ in the MSC group vs $289.3 \pm 30.3 \mathrm{~mm}^{3}$ in the vehicle group, $p=0.7285$ ) after the MCAO. There were no significant differences between the groups. Figure $4 \mathrm{G}$ summarizes these results.

\section{Quantification of EvB Extravasation}

Extravasation of EvB was quantified to evaluate the 

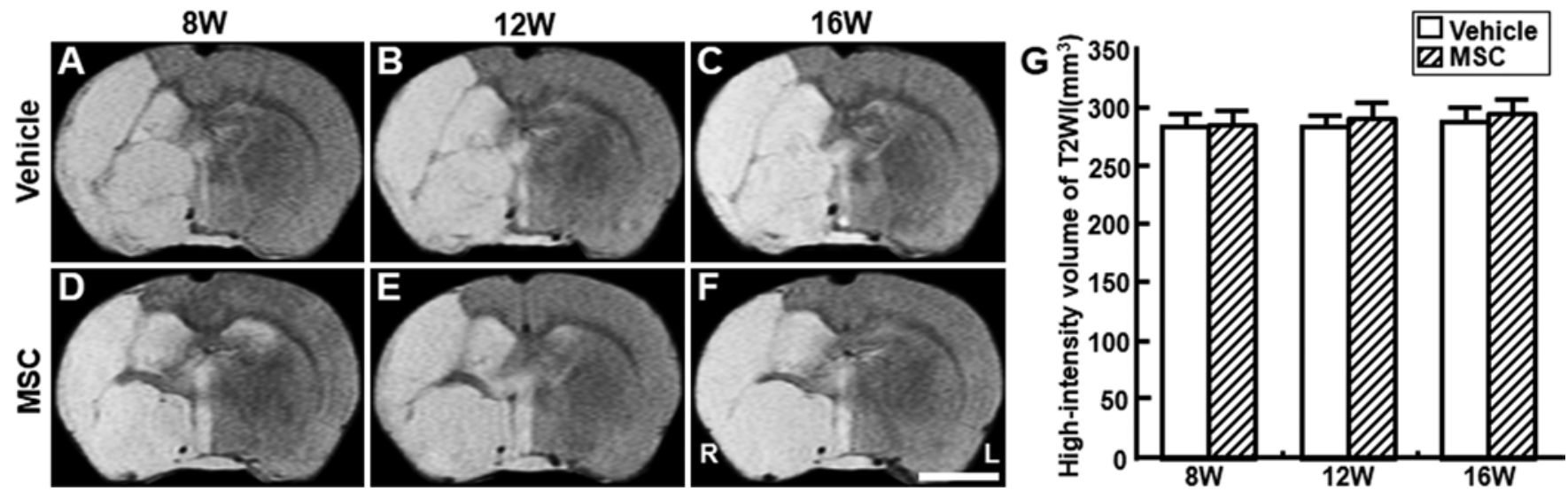

FIG. 4. Ischemic lesion volume by MRI analysis. Representative T2-weighted images (T2WIs) obtained in a rat from the vehicle group $(A-C)$ and a rat from the MSC group (D-F) at $8(A$ and $D), 12(B$ and $E)$, and $16(C$ and $F)$ weeks after MCAO. Scale bar = $4 \mathrm{~mm}$. G: Summary of lesion volume evaluated with T2Wls. There was no significant between-group difference in stroke volume. The graph presents mean values with SEMs (error bars). $L=$ left; $R=$ right.

presumptive breakdown of the BBB in rats with chronic MCAO. EvB was distributed within the brain tissue around the ischemic core in the vehicle group (Fig. 5A). However, only faint staining was observed in the brain tissue around the ischemic core in the MSC group (Fig. 5B). A significant difference was found in EvB leakage between the vehicle group and the MSC-treated group (4.28 $\pm 0.86 \mathrm{~mm}^{3}$ vs $2.00 \pm 0.51 \mathrm{~mm}^{3}$, respectively; $\mathrm{p}<0.0001$; Fig. 5C), which suggested that there was a decrease in BBB permeability of the chronically infarcted brain following MSC infusion.

\section{Profile of Microvasculature After MSC Infusion in Chronic MCAO}

Immunohistochemical analyses with RECA-1 (endothelial cells) and PDGFR- $\beta$ (pericytes) were performed to examine the microvasculature of the brain tissue around the ischemic core, at 8 weeks after MSC or vehicle infusion (Fig. 6). The microvasculature within the brain tissue around the ischemic core was examined using dual immunostaining for RECA- $1^{+}$and PDGFR- $\beta^{+}$capillary profiles (Fig. 6A-C, vehicle group; Fig. 6D-F, MSC group). The total length of endothelial cells and pericytes per square millimeter was calculated in the region of interest (see Methods). In the MSC-infused group, the length of endothelial cells (Fig. 6G, $1.99 \pm 0.23$ vs $4.64 \pm 0.27 \mu \mathrm{m} /$ $\mathrm{mm}^{2}, \mathrm{p}<0.0001$, t-test), the length of pericytes (Fig. $6 \mathrm{H}$, $0.49 \pm 0.09$ vs $\left.2.54 \pm 0.14 \mu \mathrm{m} / \mathrm{mm}^{2}, \mathrm{p}<0.0001\right)$, and the pericyte coverage rate (Fig. 6I, $24.61 \pm 2.80$ vs $55.02 \pm$ $2.11 \%, \mathrm{p}<0.0001)$ were significantly higher than in the vehicle group. Taken together, these results indicate that in chronically injured brain tissue the administration of MSCs improved BBB integrity.

\section{Discussion}

In the present study, we demonstrated in a rodent model that intravenous administration of MSCs in the chronic phase of cerebral ischemia (8 weeks after MCAO induction) improved motor function. Motor dysfunction on the treadmill test was still obvious at 2 months after the induction of MCAO, when the deficit had reached a plateau. After intravenous infusion of MSCs, motor function significantly improved beginning 1 week post-infusion. However, no recovery was observed in the vehicle-treated group. Previous studies primarily focused on acute therapeutic intervention demonstrated functional improvements. . $^{8,11,13 \text {, }}$ ${ }^{17,27}$ In the present study, therapeutic efficacy was evident,

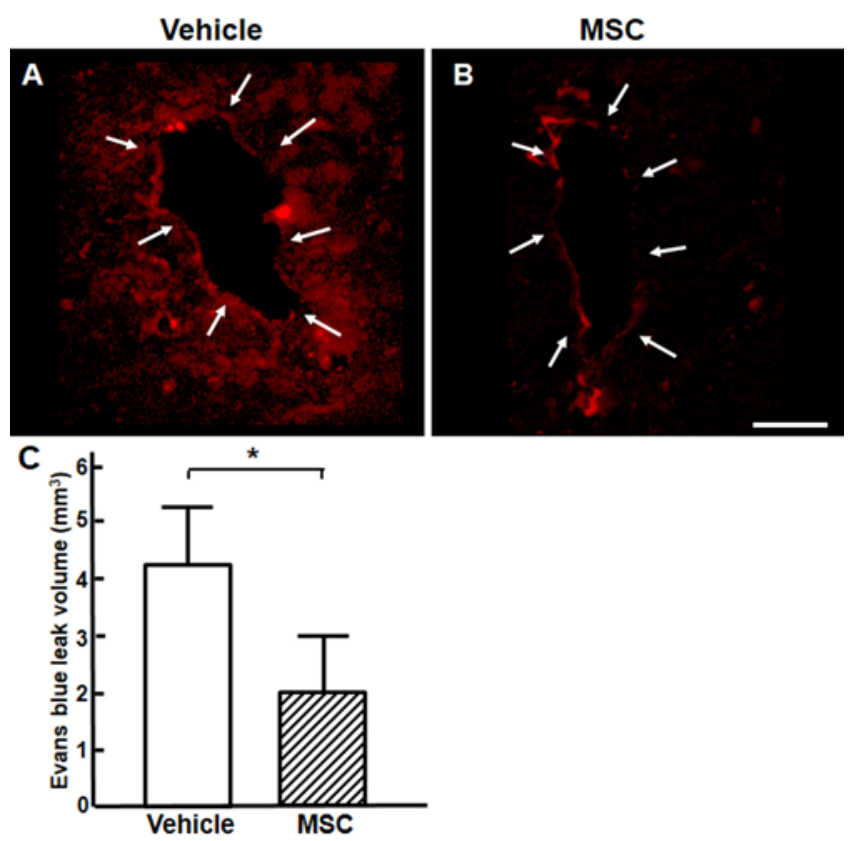

FIG. 5. Quantification of EvB extravasation. Confocal microscopic images showing EvB extravasation from microvasculature in the brain tissue around the ischemic core. EvB distribution was observed in the vehicle group (A), but only faint staining was observed in the MSC group (B). White arrows indicate microvessels. The graph (C) shows mean values of EvB-extravasated volume; the error bars indicate SEMs. Scale bar $=20 \mu \mathrm{m} .{ }^{*} p<0.0001$. 

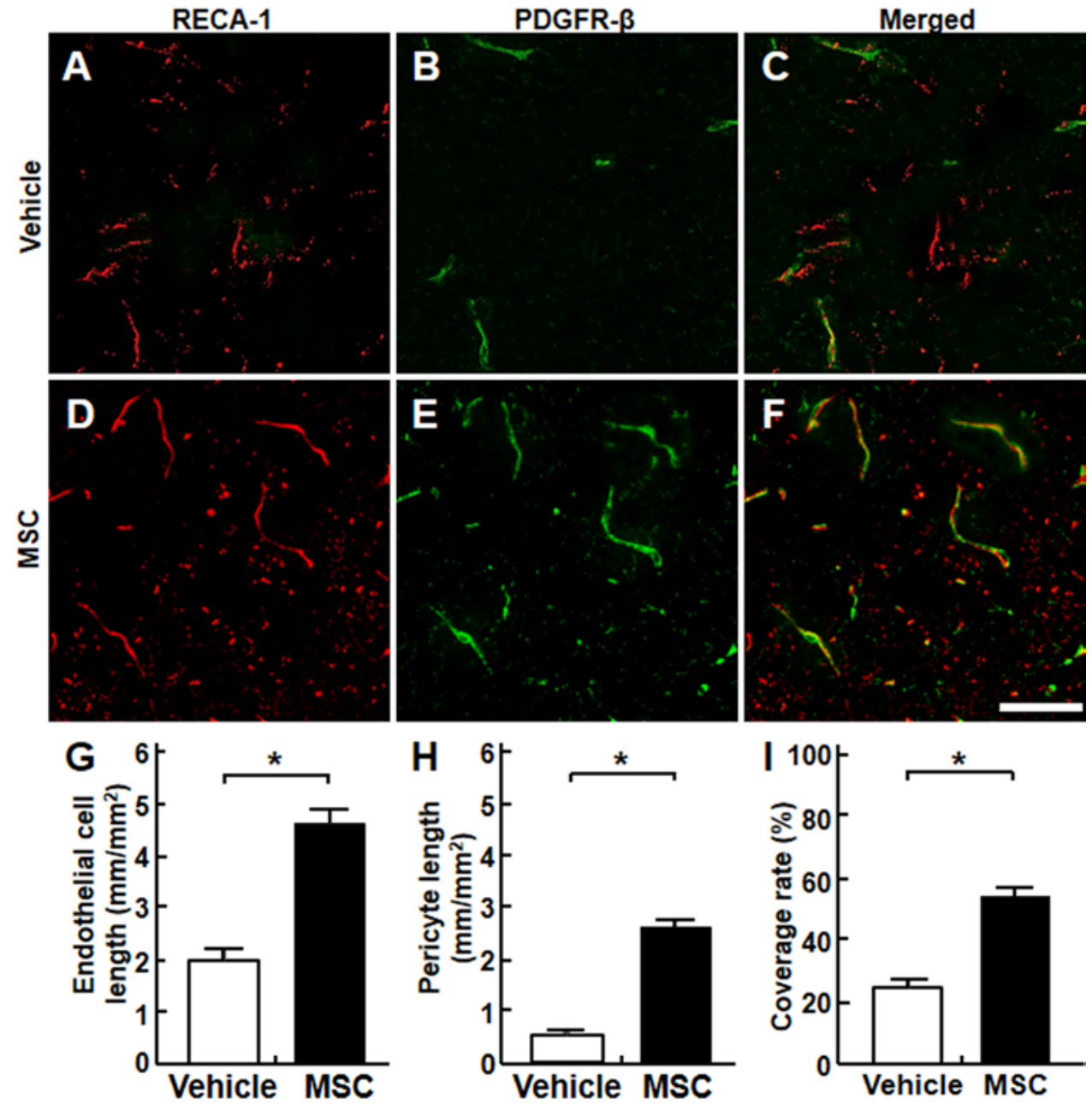

FIG. 6. Immunohistochemical profile of microvasculature. A-F: Sections of the brain around the ischemic core stained with anti-RECA-1 and anti-PDGFR- $\beta$ antibodies at 16 weeks after MCAO from vehicle-treated (A-C) and MSC-treated (D-F) rats. G-I: Mean lengths of endothelial cells $(\mathrm{G})$ and pericytes $(\mathrm{H})$ and the mean pericyte coverage rate (I). Error bars indicate SEMs. Note that a reduction of microvasculature was observed in vehicle-treated animals. Scale bar $=50 \mu \mathrm{m}(\mathrm{A}-\mathrm{F}) .{ }^{*} \mathrm{p}<0.0001$.

even when MSC infusion was delayed by 8 weeks after the MCAO induction.

The 8-week time frame after MCAO induction was chosen as a reasonable time to model "chronic" stroke in rats. Currently, knowledge about stroke is insufficient to fully define temporal categories as acute, subacute, and chronic based on biological activity. ${ }^{28}$ The operational definition could, however, be established around the stabilization of spontaneous functional recovery. ${ }^{23} \mathrm{~A}$ recent meta-analysis of preclinical studies of MSCs in ischemic stroke showed that MSCs were infused either immediately or over a period varying from 24 hours to 4 weeks; none of the 64 publications used an 8-week post-MCAO MSC administration time. ${ }^{25}$ In our stroke model, we obtained T2-weighted images 8 weeks after MCAO and excluded rats with infarcted volumes smaller than $230 \mathrm{~mm}^{3}$. H \& E staining of brain sections from animals killed at 8 weeks after MCAO induction also indicated a chronic stage of cerebral ischemia. Accordingly, the rats tested before MSC or vehicle infusion exhibited stable deficits evaluated with the treadmill stress test. Taken together, these findings suggest that the 8-week time frame after MCAO induction in the current study is appropriate to represent a persistent chronic state of cerebral infarction in rats.

Increased permeability of BBB due to cerebral ischemia has been reported to last up to $4-5$ weeks. ${ }^{1,29}$ We also observed EvB leakage 9 weeks after induction of MCAO in the vehicle-infused animals, which suggested that the BBB disruption persists for at least up to 9 weeks after MCAO in the present study. This corroborated our recent findings in traumatic spinal cord injury where vascular leakage was observed for at least 10 weeks postinjury. ${ }^{15}$ However, only faint leakage of EvB was observed in the MSC-treated groups, indicating that infused MSCs might facilitate the restoration of the disrupted BBB in this model, as it does in spinal cord injury. ${ }^{15}$ Restoration of the integrity of the BBB is important in neurological diseases, including chronic stroke. ${ }^{24}$ Recent studies indicate that pericytes control key neurovascular functions to regulate the BBB. ${ }^{2,3}$ In the present study, the vascular lengths measured from both RECA- $1^{+}$endothelial cells and PDGFR$\beta^{+}$pericytes within the lesion area around the ischemic 
core were greater in the MSC-infused group than in the vehicle-infused group. Further, the pericyte coverage rate was greater in the MSC-infused group. Taken together, the results of the EvB leakage analysis and immunohistochemical staining with RECA-1+/PDGFR- $\beta^{+}$capillary profiles demonstrated that infused MSCs could stabilize the $\mathrm{BBB}$ and reduce microvascular leakage during the chronic phase of MCAO. These results suggest that the restoration of microvasculature by infused MSCs in chronic MCAO may contribute to rapid functional improvements.

Although the precise mechanism of the beneficial effects of MSCs is not fully elucidated, previous research has demonstrated that the potential beneficial effects of MSCs in various models of central nervous system injury include induction of axonal sprouting, neovascularization, remyelination, and facilitation of synaptogenesis.9,18,27 In this study, restoration of BBB function could be an additional factor contributing to functional improvements even in the chronic stage of cerebral ischemia.

\section{Conclusions}

Our results indicated that the intravenous infusion of MSCs 8 weeks after MCAO still had beneficial effects, possibly through a combination of stabilization of the BBB and restoration of microvasculature. Thus, MSC treatment may have therapeutic value, during not only acute but also chronic cerebral ischemia.

\section{Acknowledgments}

This work was supported in part by JSPS KAKENHI grant numbers 15K10365, 16K10730, 17K10901, and 17K01513; the AMED Translational Research Network Program (JP161m0103003); and Merit Review Award 1 I01 BX003190 from the US Department of Veterans Affairs BLRD and the RRD Services (J.D.K.).

\section{References}

1. Abo-Ramadan U, Durukan A, Pitkonen M, Marinkovic I, Tatlisumak E, Pedrono E, et al: Post-ischemic leakiness of the blood-brain barrier: a quantitative and systematic assessment by Patlak plots. Exp Neurol 219:328-333, 2009

2. Armulik A, Genové G, Mäe M, Nisancioglu MH, Wallgard E, Niaudet C, et al: Pericytes regulate the blood-brain barrier. Nature 468:557-561, 2010

3. Bell RD, Winkler EA, Sagare AP, Singh I, LaRue B, Deane $\mathrm{R}$, et al: Pericytes control key neurovascular functions and neuronal phenotype in the adult brain and during brain aging. Neuron 68:409-427, 2010

4. Cramer SC: Can allogeneic stem cells improve outcomes after stroke? Lancet Neurol 16:335-336, 2017

5. Cui LL, Golubczyk D, Jolkkonen J: Top 3 behavioral tests in cell therapy studies after stroke: difficult to stop a moving train. Stroke 48:3165-3167, 2017

6. Deb P, Sharma S, Hassan KM: Pathophysiologic mechanisms of acute ischemic stroke: An overview with emphasis on therapeutic significance beyond thrombolysis. Pathophysiology 17:197-218, 2010

7. Dirnagl U, Iadecola C, Moskowitz MA: Pathobiology of ischaemic stroke: an integrated view. Trends Neurosci 22:391397, 1999

8. Honma T, Honmou O, Iihoshi S, Harada K, Houkin K, Hamada $\mathrm{H}$, et al: Intravenous infusion of immortalized human mesenchymal stem cells protects against injury in a cerebral ischemia model in adult rat. Exp Neurol 199:56-66, 2006

9. Honmou O, Onodera R, Sasaki M, Waxman SG, Kocsis JD: Mesenchymal stem cells: therapeutic outlook for stroke. Trends Mol Med 18:292-297, 2012

10. Horita Y, Honmou O, Harada K, Houkin K, Hamada H, Kocsis JD: Intravenous administration of glial cell line-derived neurotrophic factor gene-modified human mesenchymal stem cells protects against injury in a cerebral ischemia model in the adult rat. J Neurosci Res 84:1495-1504, 2006

11. Iihoshi S, Honmou O, Houkin K, Hashi K, Kocsis JD: A therapeutic window for intravenous administration of autologous bone marrow after cerebral ischemia in adult rats. Brain Res 1007:1-9, 2004

12. Kim S, Honmou O, Kato K, Nonaka T, Houkin K, Hamada $\mathrm{H}$, et al: Neural differentiation potential of peripheral blood- and bone-marrow-derived precursor cells. Brain Res 1123:27-33, 2006

13. Kocsis JD, Honmou O: Bone marrow stem cells in experimental stroke. Prog Brain Res 201:79-98, 2012

14. Liu H, Honmou O, Harada K, Nakamura K, Houkin K, Hamada H, et al: Neuroprotection by PlGF gene-modified human mesenchymal stem cells after cerebral ischaemia. Brain 129:2734-2745, 2006

15. Matsushita T, Lankford KL, Arroyo EJ, Sasaki M, Neyazi M, Radtke C, et al: Diffuse and persistent blood-spinal cord barrier disruption after contusive spinal cord injury rapidly recovers following intravenous infusion of bone marrow mesenchymal stem cells. Exp Neurol 267:152-164, 2015

16. Morita T, Sasaki M, Kataoka-Sasaki Y, Nakazaki M, Nagahama H, Oka S, et al: Intravenous infusion of mesenchymal stem cells promotes functional recovery in a model of chronic spinal cord injury. Neuroscience 335:221-231, 2016

17. Nakamura H, Sasaki Y, Sasaki M, Kataoka-Sasaki Y, Oka S, Nakazaki M, et al: Elevated brain derived neurotrophic factor (BDNF) levels in plasma but not serum reflect in vivo functional viability of infused mesenchymal stem cells after middle cerebral artery occlusion in rat. J Neurosurg Sci [epub ahead of print], 2017

18. Nakazaki M, Sasaki M, Kataoka-Sasaki Y, Oka S, Namioka $\mathrm{T}$, Namioka A, et al: Intravenous infusion of mesenchymal stem cells inhibits intracranial hemorrhage after recombinant tissue plasminogen activator therapy for transient middle cerebral artery occlusion in rats. J Neurosurg 127:917-926, 2017

19. Neumann-Haefelin T, Kastrup A, de Crespigny A, Yenari MA, Ringer T, Sun GH, et al: Serial MRI after transient focal cerebral ischemia in rats: dynamics of tissue injury, blood-brain barrier damage, and edema formation. Stroke 31:1965-1973, 2000

20. Nomura T, Honmou O, Harada K, Houkin K, Hamada H, Kocsis JD: I.V. infusion of brain-derived neurotrophic factor gene-modified human mesenchymal stem cells protects against injury in a cerebral ischemia model in adult rat. Neuroscience 136: $161-169,2005$

21. Omori Y, Honmou O, Harada K, Suzuki J, Houkin K, Kocsis JD: Optimization of a therapeutic protocol for intravenous injection of human mesenchymal stem cells after cerebral ischemia in adult rats. Brain Res 1236:30-38, 2008

22. Onda T, Honmou O, Harada K, Houkin K, Hamada H, Kocsis JD: Therapeutic benefits by human mesenchymal stem cells (hMSCs) and Ang-1 gene-modified hMSCs after cerebral ischemia. J Cereb Blood Flow Metab 28:329-340, 2008

23. Piltti KM, Salazar DL, Uchida N, Cummings BJ, Anderson AJ: Safety of human neural stem cell transplantation in chronic spinal cord injury. Stem Cells Transl Med 2:961974, 2013

24. Rosenberg GA: Neurological diseases in relation to the blood-brain barrier. J Cereb Blood Flow Metab 32:11391151,2012 
25. Sarmah D, Agrawal V, Rane P, Bhute S, Watanabe M, Kalia $\mathrm{K}$, et al: Mesenchymal stem cell therapy in ischemic stroke: a meta-analysis of preclinical studies. Clin Pharmacol Ther 103:990-998, 2018

26. Sasaki M, Honmou O, Kocsis JD: A rat middle cerebral artery occlusion model and intravenous cellular delivery. Methods Mol Biol 549:187-195, 2009

27. Sasaki Y, Sasaki M, Kataoka-Sasaki Y, Nakazaki M, Nagahama H, Suzuki J, et al: Synergic effects of rehabilitation and intravenous infusion of mesenchymal stem cells after stroke in rats. Phys Ther 96:1791-1798, 2016

28. Savitz SI, Chopp M, Deans R, Carmichael T, Phinney D, Wechsler L: Stem Cell Therapy as an Emerging Paradigm for Stroke (STEPS) II. Stroke 42:825-829, 2011

29. Strbian D, Durukan A, Pitkonen M, Marinkovic I, Tatlisumak E, Pedrono E, et al: The blood-brain barrier is continuously open for several weeks following transient focal cerebral ischemia. Neuroscience 153:175-181, 2008

30. Suzuki J, Sasaki M, Harada K, Bando M, Kataoka Y, Onodera R, et al: Bilateral cortical hyperactivity detected by fMRI associates with improved motor function following intravenous infusion of mesenchymal stem cells in a rat stroke model. Brain Res 1497:15-22, 2013

31. Toyama K, Honmou O, Harada K, Suzuki J, Houkin K, Hamada $\mathrm{H}$, et al: Therapeutic benefits of angiogenetic genemodified human mesenchymal stem cells after cerebral ischemia. Exp Neurol 216:47-55, 2009

32. Vu Q, Xie K, Eckert M, Zhao W, Cramer SC: Meta-analysis of preclinical studies of mesenchymal stromal cells for ischemic stroke. Neurology 82:1277-1286, 2014

33. Wiersma AM, Fouad K, Winship IR: Enhancing spinal plasticity amplifies the benefits of rehabilitative training and improves recovery from stroke. J Neurosci 37:10983-10997, 2017

34. Winstein CJ, Stein J, Arena R, Bates B, Cherney LR, Cramer $\mathrm{SC}$, et al: Guidelines for adult stroke rehabilitation and recovery: a guideline for healthcare professionals from the American Heart Association/American Stroke Association. Stroke 47:e98-e169, 2016

35. Zhang J, Zhang Y, Xing S, Liang Z, Zeng J: Secondary neurodegeneration in remote regions after focal cerebral infarction: a new target for stroke management? Stroke 43:17001705,2012
36. Zhang X, Zhang Q, Li W, Nie D, Chen W, Xu C, et al: Therapeutic effect of human umbilical cord mesenchymal stem cells on neonatal rat hypoxic-ischemic encephalopathy. $\mathbf{J}$ Neurosci Res 92:35-45, 2014

37. Zhao LR, Duan WM, Reyes M, Keene CD, Verfaillie CM, Low WC: Human bone marrow stem cells exhibit neural phenotypes and ameliorate neurological deficits after grafting into the ischemic brain of rats. Exp Neurol 174:11-20, 2002

38. Zheng W, Honmou O, Miyata K, Harada K, Suzuki J, Liu H, et al: Therapeutic benefits of human mesenchymal stem cells derived from bone marrow after global cerebral ischemia. Brain Res 1310:8-16, 2010

\section{Disclosures}

The authors report no conflict of interest concerning the materials or methods used in this study or the findings specified in this paper.

\section{Author Contributions}

Conception and design: M Sasaki, Kocsis, Honmou. Acquisition of data: T Namioka, A Namioka, Suzuki, Y Sasaki. Analysis and interpretation of data: M Sasaki, T Namioka, A Namioka, Kataoka-Sasaki, Honmou. Drafting the article: M Sasaki, T Namioka, A Namioka, Kocsis, Honmou. Critically revising the article: M Sasaki, T Namioka, A Namioka, Kataoka-Sasaki, Kocsis, Honmou. Reviewed submitted version of manuscript: all authors. Approved the final version of the manuscript on behalf of all authors: M Sasaki. Statistical analysis: T Namioka, A Namioka. Administrative/technical/material support: Oka, Nakazaki, Onodera, Suzuki, Y Sasaki, Nagahama. Study supervision: M Sasaki, Kataoka-Sasaki, Kocsis, Honmou.

\section{Correspondence}

Masanori Sasaki: Research Institute for Frontier Medicine, Sapporo Medical University School of Medicine, Sapporo, Hokkaido, Japan.msasaki@sapmed.ac.jp. 